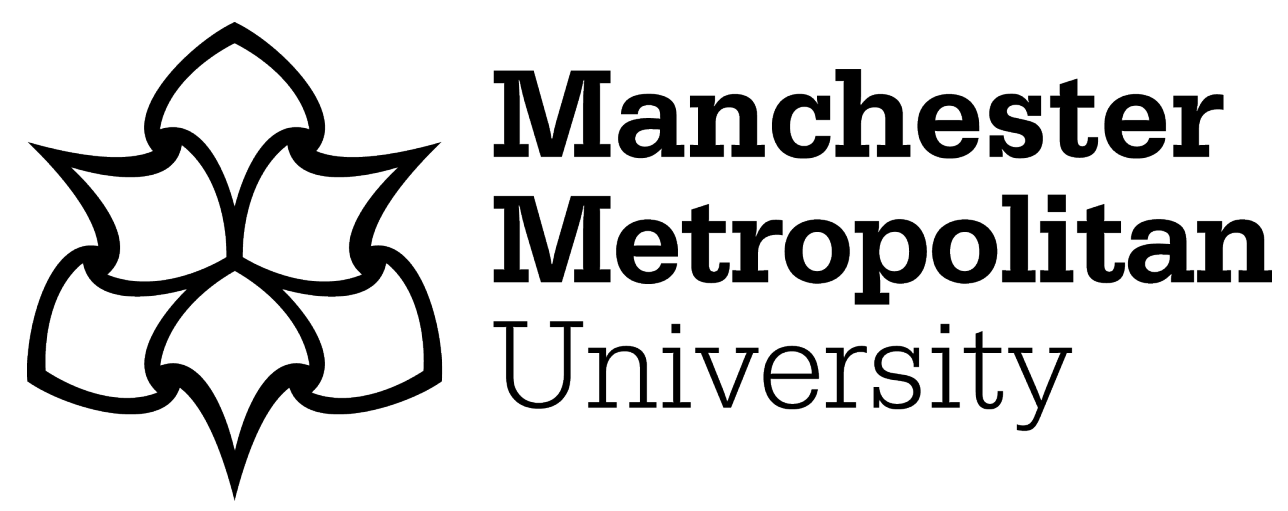

Seyedolhosseini, Atefesadat, Masoumi, Nasser, Modarressi, Mehdi and Karimian, Noushin (2019) Design and Implementation of Efficient Smart Lighting Control System with Learning Capability for Dynamic Indoor Applications. In: 2018 9th International Symposium on Telecommunications (IST), 17 December 2018 - 19 December 2018, Tehran, Iran.

Downloaded from: https://e-space.mmu.ac.uk/626760/

Publisher: IEEE

DOI: https://doi.org/10.1109/istel.2018.8661023

Please cite the published version 


\section{Design and Implementation of Efficient Smart Lighting Control System with Learning Capability for Dynamic Indoor Applications}

\author{
Atefesadat Seyedolhosseini \\ CST-LAB, Department of Electronics \\ School of Electrical and Computer \\ Engineering, College of Eng. \\ University of Tehran \\ Tehran, Iran \\ aseidolhosseini@yahoo.com
}

\author{
Nasser Masoumi \\ CST-LAB, Department of Electronics \\ School of Electrical and Computer \\ Engineering, College of Eng. \\ University of Tehran \\ Tehran, Iran \\ nmasoumi@ut.ac.ir
}

\author{
Noushin Karimian \\ School of Engineering Science \\ College of Eng., University of Tehran \\ Tehran, Iran \\ NKarimian@ut.ac.ir
}

\author{
Mehdi Modarressi \\ School of Electrical and Computer \\ Engineering, College of Eng., \\ University of Tehran, and School of \\ Computer Science, Institute for \\ Research in Fundamental Sciences \\ Tehran, Iran \\ modarressi@ut.ac.ir
}

\begin{abstract}
Accurate and efficient adjustment of luminaire's dimming level in a smart environment can be a huge challenge. Indoor lighting system as a nonlinear and time variant block, which consumes significant amount of electrical power is evaluated in this paper. In doing so, a control method is proposed to efficiently adjust luminaire's dimming level in a smart environment and to optimize energy and user's comfort level. The proposed control method takes advantages from neural network and its learning capabilities. In this research, photodetectors are placed at the work zones, where work zones can have different number of photodetectors without any increase in complexity and any adverse effect on the control system. The method is capable of adopting itself to daylight variations with high accuracy. A state machine is developed to implement the method. The method is implemented in MATLAB and lighting conditions are extracted in DIALux. Luminaire's dimming levels are determined with accuracy higher than $99 \%$. Daylight is considered as a bias to the system and thus the network does not need to be trained by any variations. In a dynamic condition, when taking into account the variation in daylight, the system mean error does not exceed $3 \%$.
\end{abstract}

Keywords-Smart Lighting, Indoor, Neural Network, Optimization, State Machine, Controller, WSN

\section{INTRODUCTION}

Artificial lighting system plays an undeniable role in the amount of consumed energy, user's comfort, health, and security. Therefore, several standards have been developed to improve lighting system performance and crucial parameters, such as light intensity and uniformity in a work area [1,2]. The effect of lighting system on human life, does not only results in new lighting technologies such as solidstate lighting, but also smart control systems to improve accuracy and system efficiency [3-5].

Presence detectors, photodetectors, light emitting diodes (LEDs), communication modules, controller equipped with a control algorithm, can all be listed as an indoor lighting system elements [7-10]. The whole system performance is significantly affected by the control algorithm implemented in the controller that determines the luminaire's dimming level according to the sensor's outputs and types of visual task carried out within the work zones [11]. The main focus of this paper is to introduce and implement control method suitable for smart indoor lighting system.

Various control methods have been proposed in the literature and each has pros and cons. One approach is to consider linear relation between photodetector's output and luminaire's dimming level; according to this approach luminaire's dimming levels are calculated based on the desired intensities at photodetector's outputs $[3,6]$. In this aspect luminaire's effect on the photodetector's outputs is modeled with light transport matrix [12]. However, each work zone may need more than one photodetectors and in some environment different zones may have common luminaires that limits the accuracy. As the lighting system is not linear and also light transport matrix cannot be calculated theoretically owing to the multiple reflections caused by object surfaces placed at the environment, a control loop should be defined to limit the system's error [3, $6]$. However, transmission rate in the network is high and control loop gain is constant $[3,6]$. Besides, owing to the theoretical limitations, number of light sensors are equal to the luminaires and they are placed adjacent to the luminaires which is not an efficient approximation [7]. Thus, linear approximation does not seem to be efficient for linear environments with multiple work zones that are dynamic and need large number of photodetectors.

The smart indoor lighting system is multiple-input multiple-output (MIMO) system as researchers mentioned in [9]. In a system with huge number of parameters that cannot be modeled with linear relations, optimization methods may be useful [13, 14]. Learning methods are mostly applicable for nonlinear and dynamic systems [14] and lighting systems can be considered as one of them. Taking the advantages of learning methods researches in [9], proposed a control methodology for indoor lighting systems. The researches claim that for static condition all sensors can be removed. However, daylight varies during daytime and the system is dynamic; besides, lighting system elements may be faulty during working phase and it should be detected by the controller. Thus, removing all the sensors 
is not a proper approach. Although the assumption considered in [9] is correct, but the input and output of the system is applied inversely that makes design unsuitable for the control system. In the research carried out in [9], zoning effect is not taken into the account, which directly affects the error of the system.

Another important issue is the placement of photodetectors at work zones, where different work zone (according to zone's dimension and light intensity variation) requires different number of photodetectors [13]; such issues are taken into account in the current paper.

Based on the idea that the lighting system is nonlinear and time variant and each indoor environment consists of multiple work zones, a control system is developed in this paper, to accurately determine luminaire's dimming level according to the desired light intensity and uniformity considered for each work zone. Neural network and learning ability is used to predict the luminaire's dimming level. The control system has the ability to detect system faults and daylight variations during daytime and adopt itself without increase in complexity. The concept of lighting system, proposed control method and test bed is discussed in section II. The proposed control system is evaluated by developed test bed and the result is presented in section III. Section IV contains a brief conclusion of the whole research.

\section{ILLUMinAtion CONTROL OF DimMABLE LIGHTING SYSTEM}

The lighting system specifications are concisely reviewed in this section and according to that, a control method suitable for indoor environment with multiple work zones is introduced. The test bed which is considered to evaluate the system performance is also described.

\section{A. $\quad$ Lighting System}

The light sources are classified into three parts; daylight, luminaires, and multiple reflections of object surfaces. The photodetector's output current can be written as (1):

$$
y_{I}=I\left(L I_{T}\right)+v_{I}
$$

where, the parameters $y_{I}, I\left(L I_{T}\right)$, and $v_{I}$ are the output current of photodetector, the equivalent current of light intensity at the sensor's position and sensor's dark current, respectively. The output current of photodetector at a fully dark place $(0 l x)$ is referred to as the dark current and should be taken into account in equations. Therefore, the equivalent light measured by the sensor can be modeled.

$$
y=L I_{T}+v
$$

In (2), the variables $y, L I_{T}$, and $v$ are the measured light intensity by sensor in lux $(l x)$, the current light intensity at the sensor's location, and the extra noise reported by the sensor. The total amount of light at the sensor's location, is the sum of three parameters, as shown in (3):

$$
L I_{T}=L I_{F}+L I_{\text {Daylight }}+M L E
$$

The total light intensity at the sensor's location $\left(L I_{T}\right)$ is sum of light intensity generated by the lighting fixtures $\left(L I_{F}\right)$, effect of day light $\left(L I_{\text {Daylight }}\right)$ and multiple reflection from the objects in the environment (MLE). The amount of reflected light from each surface is related to the transparency of objects within that environment. $M L E$ is the main parameter that results in a non-linear system, and it is due to this reason that calculating the effect of objects and multiple reflections present in a real environment is difficult. This is in addition to the fact that the effect of objects in different environment varies and as such, increases the complexity of equations. Thus, the complexity of the proposed control system is increased by considering a non-linear and time variant system. In addition, the number of luminaires and photodetectors should be equal due to the computational limitations and the reduction of accuracy for such applications.

The control method should determine all the luminaire's dimming level, according to desired light intensity, lighting uniformity, and occupancy condition of work zones. Number of photodetectors may vary based on the work zone dimensions and required accuracy in different work zones in an environment. Therefore, the number of parameters in control system is high enough to avoid theoretical modeling. Optimization methods may be useful in such systems.

In order to provide solution to such limitations, neural network $(\mathrm{NN})$ as an optimization method can be employed for such nonlinear system [14]. This type of optimization method offers an additional benefit since it can be configured in an offline mode therefore, storing the amount of power consumptions required for computational and data transfer in order to converge the desired light intensity at the sensor's location. Thus, the proposed control method that is taking advantages of $\mathrm{NN}$ is fast, accurate, and energy efficient method.

In the systems applying neural network one important issue is determination of network parameters and data required for training. In next part, detailed information in order to configure a network with optimum size for smart lighting system is introduced.

\section{B. Proposed Control System}

A novel control methodology with high accuracy and efficiency is proposed in this section. According to the standard requirements, the environment under examination is divided into multiple work zones and photodetectors are placed right at work zones [1].

Besides, each work zone may need different number of photodetectors. Presence detector is used to detect occupancy condition of work zones. Since, theoretical methods are not efficient for such system, we have used neural network in controller. Daylight variation is also taken into account in this method to efficiently determine luminaire's dimming level. The controller introduced in this paper is shown as black box as illustrated in Fig. 1.

The system with $m$ zones have $m$ occupancy sensor $\left(C_{i}\right)$ related to each zone. The system is equipped with $q$ photodetectors and according to the visual task carried out in each zone each photodetector has specific desired output 
(Des_Li). The measured output of each photodetector is calle $\bar{d}$ by (Meas_Li). occupancy condition of zones, Desired outputs of photodetectors, and measured intensities at sensors locations are considered as inputs to the control system, shown in Fig. 1. Measured outputs of photodetectors are required to track daylight variations during daytime and also to detect system (i.e. sensor's and luminaire's) faults.

The controller proposed in this paper, operate in three main modes; initialization, learning, and operational. System elements are detected in initialization mode. In learning mode, data is gathered which can be done in offline mode; hence system power consumption cab be decreased. Luminaires are dimmed individually and in groups from 0 to $100 \%$ and all sensors outputs are recorded. The speed of data gathering is limited to the speed of communication protocol and photodetectors time constant. There are various communication protocols suitable for indoor application and each may have different speed according to the operating frequency and bandwidth [13]. There is time constant to a valid response when light intensity varies at photodetector's location [3].

After data is gathered from the luminaires, neural network as an optimization block is configured. The network is initially configured with minimum size and data that is determined by the user. Network performance is evaluated, after the network is fully trained in Learning Error Check state. While network error is not in an acceptable range, the state machine changes its state to the network configuration. Initially, data amount required for network training is increased and then network size in incremented. Increase in the network size results in the higher computational power consumption which is not desired; hence, data size required for learning is increased in the first step and if the accuracy is not improved, the number of neurons and network size is increased consequently. The proposed controller is implemented by a state machine which is demonstrated in Fig. 2.

After network configuration is finished, state machine changes its state to Dim Set state. In this state, according to the desired value of each photodetector's output, the luminaire's dimming level is delivered by the neural network. The control system goes to the standby mode and system error is calculated every 15 minutes in detection error source state. The error is the difference between measured and desired value of intensity at each sensor's location. If the network error is in the acceptable range according to the visual task in each zone, the state machine changes its state to the standby state. If the error is not in a desired region, error source is detected in the controller. If the error causes faults in system element, user alarm is activated to remove faulty element and if the error increased due to the daylight variation, daylight effect is added to the system and new desired value for each sensor is calculated.

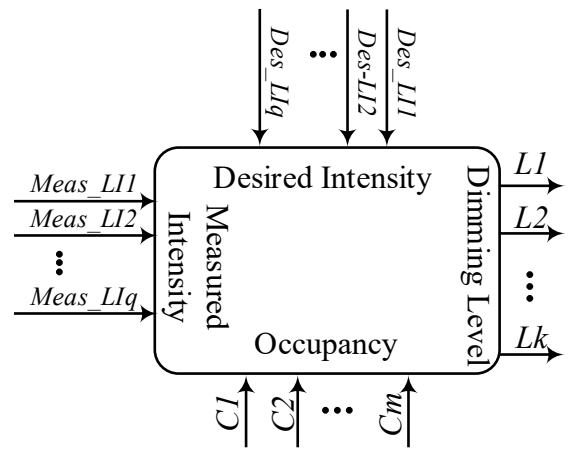

Fig. 1. Lighting system inputs and outputs

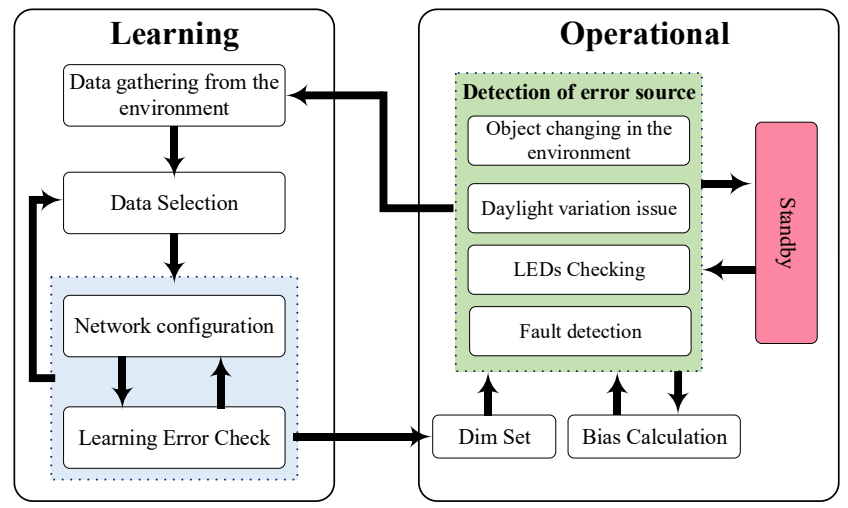

Fig. 2. Proposed control states in order to configure neural network, detect faults, and bias calculations

As mentioned, daylight variation is taken into account in the proposed controller. Daylight is considered as a bias to the system (4). The new desired values of photodetector's outputs are calculated and it is applied to the system as shown in (5):

$$
\begin{gathered}
\text { Bias_Li } i_{i}=\text { Des_Li } i_{i}-M e a s_{-} L i_{i} \\
N e w_{-} \text {Des_Li } i_{i}=B i a s_{-} L i_{i}+\text { Des_Li } i_{i}
\end{gathered}
$$

where in (4) and (5), Bias_Li $L i_{i}$ is the bias of the $i^{\text {th }}$ sensor output which is added to the $\bar{D} e s_{-} L i_{i}$ (desired intensity of $i^{t h}$ sensor output) to formulate the new desired value. Meas_Li $i_{i}$ is the measured value of $i^{\text {th }}$ photodetector which may vary from the desired value. The difference between current value of photodetector and desired value is considered as bias to system. Thus, new values of desired intensities are calculated and considered as an input to the neural network.

In this part, accurate and fast response of the control method is introduced which is suitable for dynamic indoor systems. Photodetectors are placed at the work zones and each work zone has more than one photodetector. In order to optimize the system, neural network is applied and a state machine is proposed to regulate the system.

\section{Test Bed}

A test bed is considered to evaluate the performance of the proposed system. A room consisting of five work zones is considered in the system under examination. Each work 
zone receives different daylight effect. The specifications such as room dimensions, luminaire types, room objects and reflection ratio of them, window and its location, daytime, and geographical specifications of the office are all considered by the user as the parameters for the test bed, and in order to evaluate the proposed control method these parameters are all implemented in DIALux.

Dimming level of each luminaire is entered as an input and the minimum, maximum, and average light intensity of various points of work zone is reported as an output in DIALux. Besides, only the first reflections are taken into account to evaluate the control system. The $3 \mathrm{D}$ view of implemented test bed in DIALux software is shown in Fig. 3.

As shown in Fig. 3 and reported in Table I, the room contains 12 luminaires ( DIAL 11 R2600/158 P8 type) which can $\operatorname{dim}$ from 0 to $100 \%$ and 4 work zones. The window is placed to enter the daylight to the indoor environment.

The work zones in the test bed are indicated by desks which are identified in Fig. 4 by green rectangles in the floor plan. Light intensity at the determined areas are the desired values, which based on the work done in the area should be controlled.

The effect of daylight is reported in Table II, where for each zone, the maximum, minimum, and average measured light intensity when all luminaires are off, are reported. The work zone located adjacent to the window (Zone 1) senses more light compared to the other zones. Zone number 2 and 3 are located far from the window and therefore the reported light intensity is at the lowest values.

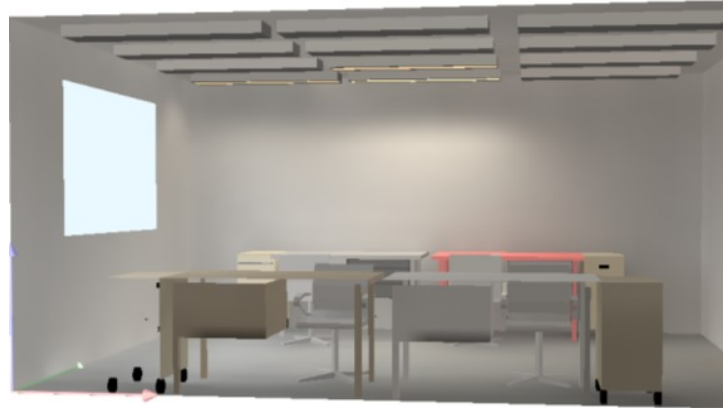

Fig. 3. Implemented test bed at DIALux software to evaluate the proposed controller

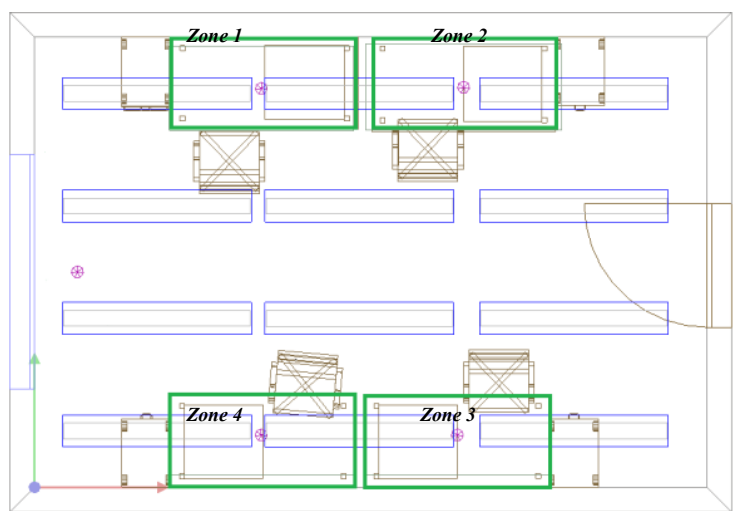

Fig. 4. Floor plan of implemented test bed with 4 work zones with work areas identified as green rectangles
TABLE I. DETAILED INFORMATION ABOUT THE CONSIDERED TESTBED FOR SiMULATIONS VERIFICATIONS

\begin{tabular}{|l|l|}
\hline \multicolumn{1}{|c|}{ Parameter } & \multicolumn{1}{c|}{ Value } \\
\hline Room Area $\left(\mathrm{m}^{2}\right)$ & 20 \\
\hline Room Height (m) & 2.74 \\
\hline Wall Reflection (\%) & 20 \\
\hline Number of Zones & 4 \\
\hline Number of Luminaires & 12 \\
\hline Work Zone Area (m $\left.{ }^{2}\right)$ & $1.52 \times 0.75$ \\
\hline Work Zone Height (m)* & 0.76 \\
\hline Number of Sensors Per Zone & 5 \\
\hline $\begin{array}{l}\text { Average Daylight } \\
\text { Deviation** }\end{array}$ & $\begin{array}{l}\mathrm{Z} 1=35 \%, \mathrm{Z} 2=61 \% \\
\mathrm{Z} 3=36 \%, \mathrm{Z}=35 \%\end{array}$ \\
\hline Sky Type & Overcast \\
\hline
\end{tabular}

* This is reported when all luminaires are off from 8a.m. to 8 p.m. Deviation from maximum received intensity is stored and average deviation from the maximum is then reported. Deviatioin $=$ $((\max$-node $) / \max ) * 100$

TABLE II. The MaXimum, Minimum, AND AVERage Measured LIGHT INTENSITY OF VARIOUS ZONES IN TESTBED WHEN ALL LUMINAIRES OFF

\begin{tabular}{|l|l|l|l|l|}
\hline \multicolumn{1}{|c|}{ Zalue } & Zone 1 & Zone 2 & Zone 3 & Zone 4 \\
\hline Maximum $(l x)$ & 196 & 91 & 94 & 220 \\
\hline Minimum $(l x)$ & 131 & 75 & 80 & 139 \\
\hline Average $(l x)$ & 284 & 115 & 107 & 357 \\
\hline
\end{tabular}

As it is reported in Table III, sensor's location significantly affects not only the accuracy but also the power consumption. The variation in sensor's output is more than $70 \%$ when they are placed adjacent to the luminaires in the test bed shown in Fig. 3. As it is reported, ceiling mounted sensors receive lower amount of light compared to the work zone mounted ones. Thus, the power consumption for the ceiling mounted sensor is higher in comparison to those placed at the work zones.

TABLE III. DIFFERENCE BETWEEN MEASURED VALUE OF PHOTODETECTORS AT DifFERENT LOCATIONS WHEN LUMINAIRES 4 AND 7 ARE FULLY ON

\begin{tabular}{|l|l|}
\hline Location & Measured Intensity \\
\hline \multirow{4}{*}{ Zone \#1 } & 968.8 \\
\cline { 2 - 2 } & 839.6 \\
\cline { 2 - 2 } & 1054.9 \\
\cline { 2 - 2 } & 828.8 \\
\cline { 2 - 2 } & 947.2 \\
\hline \multirow{4}{*}{ Ceiling } & 204.5 \\
\cline { 2 - 2 } & 172.2 \\
\hline
\end{tabular}

\section{Simulation RESULTS}

The test setup described in section II, is used to evaluate the control system developed in this paper. The proposed 
controller is implemented in MATLAB and lighting conditions are evaluated in DIALux.

The test bed considered in this paper, has 12 luminaires which means that the control system has 12 outputs. In addition, the inputs to the system are related to the number of sensors in the system. The number of sensors considered for each zone is 5 . Therefore, the controller should receive 20 inputs for the desired light intensities at each sensor's location and 20 inputs for measured values of photodetectors and 5 inputs for occupancy condition of each work zone.

As shown in Fig. 2, the network is initially configured with minimum number of neuron and data. If the network error is higher than desired region, the data size required for training is initially increased and then the number of neuron is incremented afterward. This is due to the fact that an increase in the number of neurons results in higher computational power [14]. A feedforward neural network is, therefore, implemented using MATLAB.

Neural network is primarily configured with 5 neurons and 500 data sets for training; since network error does not converge to the desired region, even by an increase of $1 \%$ in data set. The working number of neurons are then increased to 11 step by step, results of such network configuration is shown in Fig. 5. As it can be seen, the network mean error is below $1 \%$ for 3000 training data sets. Additional 300 data sets which are completely different from the ones used to train is applied for testing to evaluate the network for new data. The error histogram of test data after network configuration when different data sizes is used to train the network is shown in Fig. 5.

The error histogram the neural network in training with 3000 data sets for training is shown in Fig. 6. The error histogram is related for the feedforward network with 11 neurons.

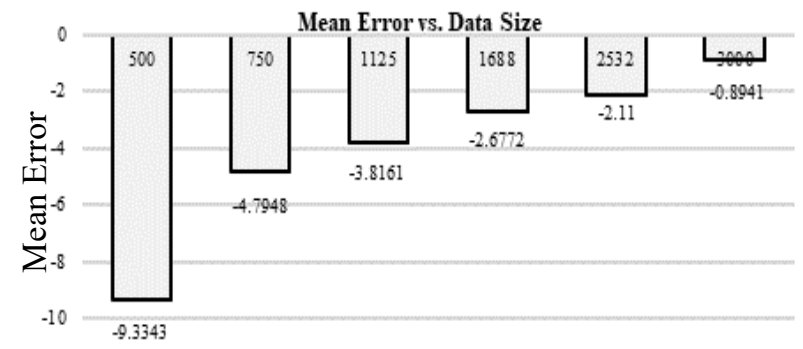

Fig. 5. Mean error variation vs. data size considered for training a feedforward neural network with one hidden layer and 11 neurons

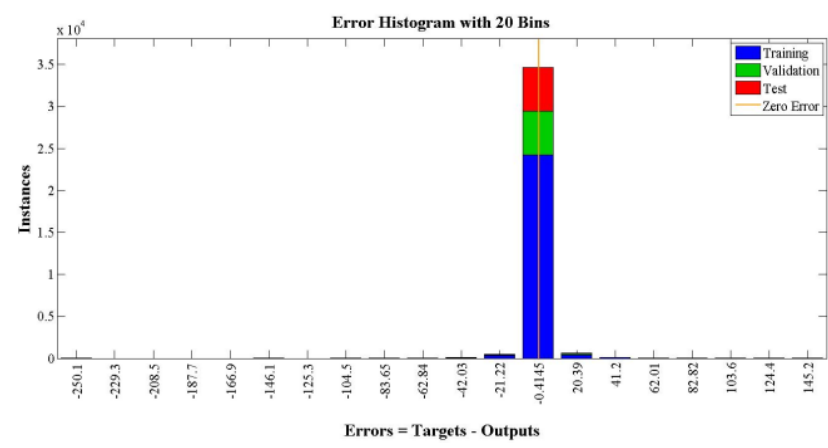

Fig. 6. The error histogram of feedforward network contains one hidden layer and 11 neurons and 3000 data sets considered for training

Daylight variation as an important factor and a bias to the system is considered in the proposed control method, as described earlier. The difference between the desired value of sensor's outputs and measured ones are considered as bias and are added to the desired value of the sensor's outputs and then applied to the employed neural network.

To evaluate the method, different daytime and required lighting condition is simulated. In each case, daylight variation is considered as bias to desired intensity as mentioned in (4) and (5). Thus, dimming level of luminaires are delivered by the neural network. Finally, the difference between the light intensity at photodetectors locations and initial desired value of zone 1 sensors is calculated as error and the results are shown in Fig. 7, the maximum error is below 3\%, which compared to the results from similar research that have been higher than $70 \%$, is an achievement. Daylight variation and proposed method is evaluated for the zone 1 , since it receive maximum effect from the daylight. It means that zone 1 reports worst case error which is below $3 \%$ and it is in the desired area.

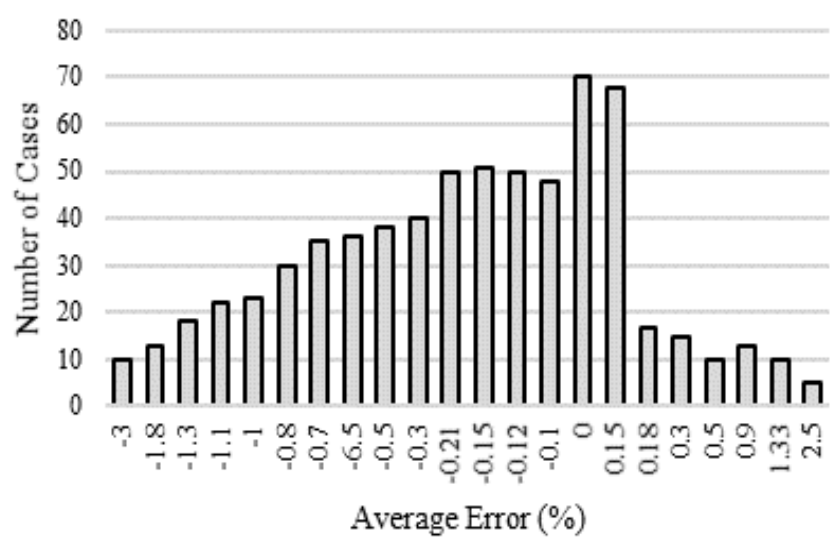

Fig. 7. The percentage of error variation between desired outputs of sensors and measured one in consideration of daylight as a bias in 670 different cases for work zone \#1

\section{CONCLUSION}

In this paper, a smart lighting control system suitable for an indoor environment with multiple luminaires and work zones and taking into account the daylight variation, is proposed. The proposed control strategy is smart, accurate, and energy efficient, since it considers the nonlinear and time variant effects of real lighting system. A state machine 
is developed to advance the performance of controller. The controller performance is improved by taking advantages of learning methods. In doing so, neural network has been applied to add the learning capability. Daylight variation during the daytime is taken into account in the proposed controller. The lighting conditions are extracted from DIALux and the proposed controller is implemented in MATLAB. The obtained error from the controller is below $1 \%$ for the considered test bed. In a dynamic system, when considering daylight variation, the system error is found to increase to $3 \%$, which still is lower than the proposed methods and results seen in literature.

\section{REFERENCES}

[1] Light and Lighting - Lighting of Work Places - Part I: Indoor Work Places, European Standard, EN 12464-1, 2011.

[2] Lighting of Work Places - Part II: Outdoor Work Places, European Standards, EN 12464-2, 2014.

[3] S. Afshari, S. Mishra, A. Julius, F. Lizarralde, J. D. Wason, J. T. Wen, "Modeling and Control of Color Tunable Lighting Systems," Elsevier Journal on Energy and Building, no. 68, pp. 242-253, 2014.

[4] S. Borile, A. Pandharipande, D. Caicedo, A. Cenedese, and L. Schenato, "An Identification Approach to Lighting Control," European Control Conference (ECC), 2016.

[5] Z. Kaleem, T. M. Yoon, and Ch. Lee, "Energy Efficient Outdoor Light Monitoring and Control Architecture Usign Embedded Systems," IEEE Embedded Systems Letters, vol. 8, no. 1, Mar. 2016.

[6] S. Afshari and S. Mishra, "A Plug-and-Play Realization of Decentralized Feedback Control for Smart Lighting Systems," IEEE Trans. On Control Systems Technology, vol. 24, no. 4, Jul. 2016.

[7] S. Li, A. Pandharipande, and F. M. J. Willems, "Daylight Sensing LED Lighting System," IEEE Sensors Journal, vol. 16, no. 9, May 2016.

[8] D. Caicedo and A. Pandharipande, "Distributed Illumination Control With Local Sensing and Actuation in Networked Lighting Systems," IEEE Sensors Journal, vol. 13, no. 3, Mar. 2013.

[9] D. Tran and Y. Kh. Tan, "Sensorless Illumination Control of A Networked LED-Lighting System Using Feedforward Neural Network," IEEE Trans. On Industrial Electronics, vol. 61, no. 4, Apr. 2014

[10] F. Viani, A. Polo, P. Garofalo, N. Anselmi, M. Salucci, and E. Giarola, "Evolutionary Optimization Applied to Wireless Smart Lighting in Energy-Efficient Museums, "IEEE Sensors Journal, vol. 17 , no. 5, 2017

[11] I. Chew, V. Kalavally, Ch. P. Tan, and J. Parkkinen, "A SpectrallyTunable Smart LED Lighting System with Closed-Loop Control," IEEE Sensnors Journal, vol. 16, no. 11, 2016.

[12] J. Foely, A. V. Dam, and S. K. Feiner. Computer Graphics: Principles and Practice in C. $2^{\text {nd }}$ Edition, Addison-Wesley Professional, 1999.

[13] A. Pandharipande and D. Caicedo, "Smart Indoor Lighting Systems with Luminaire-Based Sensing: A Review of Lighting Control Approaches," Elsevier Journal on Energy and Buildings, pp. 369377, 2015.

[14] I. W. Sandberg, J. T. Lo, C. L. Fancourt, J. C. Principe, S. Katagiri, and S. Hayakin, "Nonlinear Dynamical Systems, Feedforward Neural Network Perspectives" John Wiley \& Sons, 2001. 